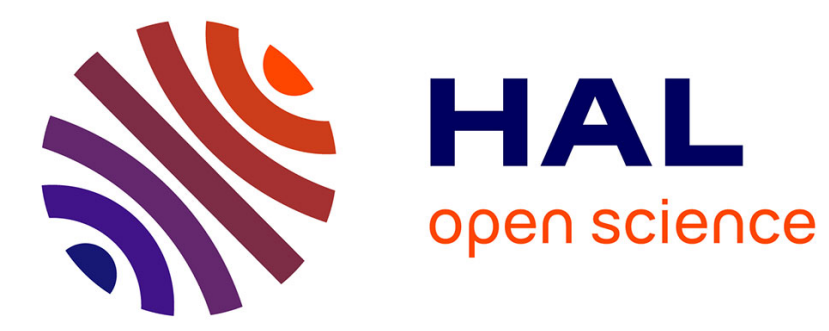

\title{
Automatic determination of wind profiles with Generalized SCIDAR
}

\author{
Jean-Louis Prieur, Rémy Avila, Gérard Daigne, Jean Vernin
}

\section{To cite this version:}

Jean-Louis Prieur, Rémy Avila, Gérard Daigne, Jean Vernin. Automatic determination of wind profiles with Generalized SCIDAR. Publications of the Astronomical Society of the Pacific, 2004, 116, pp.778789. hal-02459905

\section{HAL Id: hal-02459905 https://hal.science/hal-02459905}

Submitted on 29 Jan 2020

HAL is a multi-disciplinary open access archive for the deposit and dissemination of scientific research documents, whether they are published or not. The documents may come from teaching and research institutions in France or abroad, or from public or private research centers.
L'archive ouverte pluridisciplinaire HAL, est destinée au dépôt et à la diffusion de documents scientifiques de niveau recherche, publiés ou non, émanant des établissements d'enseignement et de recherche français ou étrangers, des laboratoires publics ou privés. 


\title{
Automatic determination of wind profiles with Generalized SCIDAR
}

\author{
J.-L. Prieur \\ UMR 5572 d'Astrophysique, Observatoire Midi-Pyrénées - CNRS, 14, Avenue Edouard \\ Belin, 31400 Toulouse, France$$
\text { jean-louis.prieur@obs-mip.fr }
$$$$
\text { R. Avila }{ }^{1}
$$ \\ Centro de Radioastronomía y Astrofísica, UNAM, A.P. 72-3 (Xangari), 58089 Morelia \\ Michoacán, México \\ r.avila@astrosmo.unam.mx \\ G. Daigne
}

UMR 5804, Observatoire Aquitain des Sciences de l'Univers, BP 89, 33270 Floirac, France daigne@obs.u-bordeaux1.fr

and

J. Vernin

UMR 6525 Laboratoire Universitaire d'Astrophysique de Nice, Université de Nice Sophia Antipolis - CNRS, Parc Valrose, 06108 Nice Cédex 2, France

$$
\text { jean.vernin@unice.fr }
$$

Received accepted

\footnotetext{
${ }^{1}$ On leave to Instituto de Astronomía, UNAM, Mexico
} 


\begin{abstract}
We present an iterative method for deriving wind profiles from Generalized SCIDAR measurements, which can work in a non-supervised mode. It is an extension of our CLEAN-based method previously developed for $C_{\mathrm{N}}^{2}$ profile determination. The algorithm is based on a morphological analysis of the cross-correlation function of consecutive scintillation irradiance frames, with the introduction of some knowledge from the $C_{\mathrm{N}}^{2}$ profiles, which are determined from the autocorrelation of those frames. This method was successfully tested on data from the site testing observations made at San Pedro Mártir in 2000, even on the most difficult cases.
\end{abstract}

Subject headings: Atmospheric effects - Site testing - Turbulence - Methods: data analysis - Techniques: image processing

\title{
1. Introduction
}

The SCIDAR method (SCIntillation Detection And Ranging) was proposed by Rocca et al. (1974) to characterize the atmospheric turbulence that perturbs astronomical observations. This method is based on the analysis of the autocorrelation of irradiance images of a binary star, in the pupil plane. It allows the determination of (i) vertical profiles of the refractive-index structure $C_{\mathrm{N}}^{2}(h)$, which characterizes the strength of the optical turbulence, and (ii) the velocities $\boldsymbol{V}$ of the turbulent layers ${ }^{2}$.

The original SCIDAR method did not allow the determination of the turbulence of layers located close to the ground level, or inside the dome. To allow such a determination,

\footnotetext{
${ }^{2}$ In this paper, two-dimensional vectors will be noted in bold
} 
Fuchs et al. (1998) proposed to shift the (virtual) plane of analysis a few km below the pupil plane. Avila et al. (1997) implemented this method on a telescope. This extension of the SCIDAR method is known as Generalized SCIDAR, and will be noted GS in the following of this paper.

In the last few years, extensive observation campaigns have been performed with GS to study the turbulence above astronomical observatories (Avila et al., 1998; Klückers et al., 1998; Vernin et al., 2000,; Avila et al., 2004; Mckenna et al., 2003; Chun et al., 2002; García-Lorenzo, 2003). Although profiles of $C_{\mathrm{N}}^{2}(h)$ are routinely calculated from those observations, with Maximum Entropy methods (Vernin, 1992; Klückers et al., 1998) or CLEAN (Prieur et al., 2001), few papers presenting wind profiles have been published until now (Klückers et al., 1998; Avila et al., 2001; Vernin et al., 2000; Avila et al., 2004). The main reason is that wind parameters are rather difficult to retrieve from SCIDAR (or GS) data. To our knowledge, only very tedious interactive programs exist to do so (Avila et al., 2001). We present here the results of our investigations in order to perform an automatic determination of wind profiles that can be run in batch mode on a large amount of data.

\section{The SCIDAR method}

The SCIDAR technique has been the subject of many papers (e.g. Rocca et al., 1974; Vernin \& Azouit, 1983; Caccia et al., 1987; Avila et al., 1997, Klückers et al., 1998, Prieur et al., 2001). Here we only present the guidelines of the method in order to introduce the quantities useful for this paper.

In this section we shall assume that the observations are performed at the zenith. For non-zero zenithal angles $\gamma$, the altitudes $h$ should be replaced by $h \cos ^{-1}(\gamma)$ in all equations. 


\subsection{Principle of the $C_{\mathrm{N}}^{2}(h)$ measurements}

Let us first consider the observation of a single star in the presence of a single thin turbulent layer at altitude $h$ above the ground, with a thickness $\delta h$, and a refractive-index structure coefficient of $C_{\mathrm{N}}^{2}(h)$. This layer will introduce phase fluctuations in the light path that will generate intensity fluctuations, i.e., scintillation, at the level of the ground, whose covariance is $\mathcal{C}(\mathbf{r}, h) \times C_{\mathrm{N}}^{2}(h) \delta h$. When assuming that the phase fluctuations produced by the layer have a Kolmogorov spectrum, it can be shown that:

$$
\mathcal{C}(\mathbf{r}, h)=0.66 \pi^{1 / 3} \lambda^{-2} \int \mathrm{d} \mathbf{f} f^{-11 / 3} \sin ^{2}\left(\pi \lambda h f^{2}\right) \exp (-2 \mathrm{i} \pi \mathbf{f} \cdot \mathbf{r})
$$

where the symbol $\mathbf{f}$ represents the two-dimensional spatial frequency, and $f$ is its modulus. Hence the contribution $\sigma_{I}^{2}(h)$ of this layer to the total scintillation variance $\sigma_{I}^{2}$ at the ground level is given by:

$$
\sigma_{I}^{2}(h) \equiv \mathcal{C}(0, h) \int_{h-\delta h / 2}^{h+\delta h / 2} C_{\mathrm{N}}^{2}(h) \mathrm{d} h=19.12 \lambda^{-7 / 6} h^{5 / 6} J(h)
$$

where $J(h)$ is the optical turbulence factor of this layer:

$$
J(h)=\int_{h-\delta h / 2}^{h+\delta h / 2} C_{\mathrm{N}}^{2}(h) \mathrm{d} h
$$

In the case of a double star whose components have an angular separation $\boldsymbol{\rho}$, the scintillation pattern will be duplicated at the level of the ground, with a horizontal distance $\boldsymbol{\rho} h$ between the two patterns. Hence, profiles of $C_{\mathrm{N}}^{2}(h)$ can be easily derived from the analysis of the mean spatial autocorrelation function of short exposure-time images of the scintillation pattern produced by a double star. This is the principle of the SCIDAR technique.

In classical SCIDAR, the telescope pupil is imaged onto the detector, which makes the technique insensitive to turbulence close to the ground, because the scintillation variance is proportional to $h^{5 / 6}$ (cf. Eq. 2). In GS, the plane of the detector is made the conjugate of a 
plane at a distance $h_{\mathrm{gs}}$ (the analysis plane), which lies a few kilometers below the telescope pupil (i.e. $\left.h_{\mathrm{gs}}<0\right)$. In this case the turbulence near the ground, including that in the telescope dome, becomes detectable because the distance relevant for scintillation produced by a turbulent layer at an altitude $h$ is now $H=h-h_{\mathrm{gs}}$, which is indeed the distance between the phase screen, and the plane of observation (cf Fig. 1). Therefore, Eqs. 1 and 2 remain valid when simply replacing $h$ by $H$.

As the different turbulent layers are statistically independent, the contribution of each one is added, and the total theoretical autocorrelation function can be written as (cf. Roddier, 1981):

$$
C^{* *}(\mathbf{r})=\int_{-h_{\mathrm{gs}}}^{+\infty} \mathrm{d} h C_{\mathrm{N}}^{2}(h)[a \mathcal{C}(\mathbf{r}, H)+b \mathcal{C}(\mathbf{r}-\boldsymbol{\rho} H, H)+b \mathcal{C}(\mathbf{r}+\boldsymbol{\rho} H, H)]
$$

The factors $a$ and $b$ of Eq. (4) are given by

$$
a=\frac{1+\alpha^{2}}{(1+\alpha)^{2}} \quad \text { and } \quad b=\frac{\alpha}{(1+\alpha)^{2}}, \quad \text { with } \quad \alpha=10^{-0.4 \Delta m},
$$

where $\Delta m$ is the magnitude difference of the double star.

Hence, all the information needed to retrieve $C_{\mathrm{N}}^{2}(h)$ is contained in a radial section of $C^{* *}(\mathbf{r})$ along the double star separation. Furthermore, it is convenient to isolate the satellites from the central peak, because in the latter the contribution of each layer is indistinguishable from that of the others, as they are added, and it contains the uncorrelated noise. The result of the radial section ( $x$ direction) and of the isolation of say the peak on the left hand side located at $x_{\text {left }}$, for experimental data, can be expressed as:

$$
B_{\mathrm{gs}}^{* *}(x)=\int_{-h_{\mathrm{gs}}}^{+\infty} \mathrm{d} h K\left(x-x_{\text {left }}, H\right) C_{\mathrm{N}}^{2}(h)+N(x) .
$$

$N(x)$ is the noise, and the kernel $K\left(x-x_{\text {left }}, H\right)$ is a radial section of $b \mathcal{C}(\mathbf{r}-\boldsymbol{\rho} H, H) * S(\mathbf{r})$, where $S(\mathbf{r})$ is the autocorrelation of the impulse response (PSF) of the detector. The determination of $C_{\mathrm{N}}^{2}(h)$ is thus an inverse problem, obeying Eq. (6), which is an equation 
of Fredholm type. It can be solved by various numerical methods, for instance using a maximum entropy algorithm (e.g., Vernin, 1992) or CLEAN (e.g., Prieur et al., 2001)

Vernin \& Azouit (1983) showed that $\Delta \mathcal{R}(h)$ the equivalent radius (width at half maximum) of a correlation peak for a given layer at altitude $h$ is proportional to $\sqrt{\lambda\left(h-h_{\mathrm{gs}}\right)}$. The proportionality constant has been determined by Prieur et al. (2001) to be equal to 0.78 , so that:

$$
\Delta \mathcal{R}(h)=0.78 \sqrt{\lambda\left(h-h_{\mathrm{gs}}\right)}
$$

\subsection{Principle of wind velocity $\mathbf{V}(h)$ measurements}

In the following, we shall assume that the turbulent structures are carried by the mean wind without deformation. This assumption is known as Taylor hypothesis, and is valid for short enough time intervals. In this case, the scintillation pattern produced by a layer at altitude $h$, where the mean (horizontal) wind velocity is $\mathbf{V}(h)$, moves on the analysis plane a distance $\mathbf{V}(h) \Delta t$ in a time $\Delta t$. Hence, $\mathbf{V}(h)$ can be determined by analyzing the cross-correlation of pairs of scintillation images taken at times separated by $\Delta t$. As in the case of the autocorrelation (cf. Sect 2.1), the presence of a turbulent layer at height $H$ produces a triplet in the cross-correlation function, with a separation of $\pm \boldsymbol{\rho} H$ between the central peak and the satellites. But here the central peak is no longer situated at the origin: it is located at the point $\mathbf{r}=\mathbf{V}(h) \Delta t$. In the case of multiple layers, by analogy with Eq. (4), the cross-correlation can be written as:

$$
\begin{gathered}
C_{\mathrm{c}}^{* *}(\mathbf{r}, \Delta t)=\int_{-h_{\mathrm{gs}}}^{+\infty} \mathrm{d} h C_{\mathrm{N}}^{2}(h)\left[a \mathcal{C}_{\mathrm{c}}(\mathbf{r}-\mathbf{V}(h) \Delta t, H)+b \mathcal{C}_{\mathrm{c}}(\mathbf{r}-\mathbf{V}(h) \Delta t-\boldsymbol{\rho} H, H)\right. \\
\left.+b \mathcal{C}_{\mathrm{c}}(\mathbf{r}-\mathbf{V}(h) \Delta t+\boldsymbol{\rho} H, H)\right] .
\end{gathered}
$$

$\mathcal{C}_{\mathrm{c}}$ differs slightly from $\mathcal{C}(\mathrm{Eq} .4)$ because of a certain amount of temporal de-correlation of the scintillation (partial failure of the Taylor hypothesis), and a possible fluctuation of 


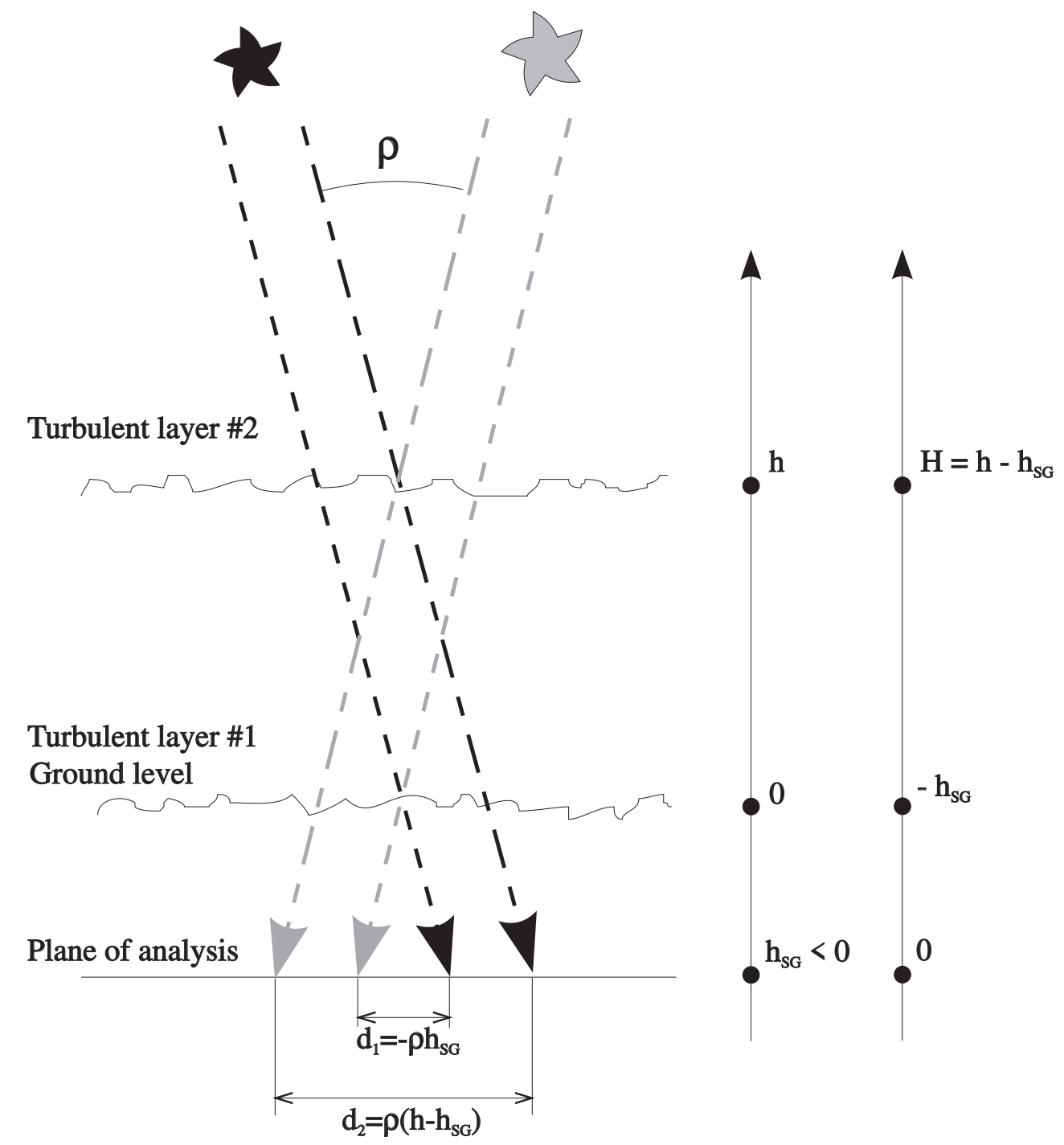

Fig. 1. - Principle of the generalized SCIDAR. 
$\mathbf{V}(h)$ during the integration time. Those two effects reduce the amplitude of the scintillation covariance and widen it, respectively (Caccia et al., 1987). In the current implementation of our method we have assumed that $\mathcal{C}_{\mathrm{c}}(\mathbf{r}, H) \propto \mathcal{C}(\mathbf{r}, H)$ and neglected the (small) width increase. The absence of artefacts in the residual maps we have obtained so far indicates that this assumption was valid for the data we have processed with our method.

\subsection{Sensitivity of the method}

Experimentally, we are limited to a finite pupil size, and the intensity of $B_{\mathrm{gs}}^{* *}(x)$ of Eq. 6 is modulated (i.e., multiplied) by $P(x)$, the autocorrelation of the pupil. We can thus expect that the noise increases with $x$, the abscissa of the secondary peak. As can be seen in Fig. 1, this quantity is proportional to $\boldsymbol{\rho}$, the angular separation of the binary and to $h_{S G}$, the distance of the analysis plane from the ground level. Hence for GS observations, one should select binaries appropriately to obtain a good compromise between large values of $x$ to allow a good separation of turbulent layers with a high resolution in altitude, and small values of $x$ to reduce the noise.

When neglecting the read-out noise, Tokovinin (1997) has shown that the statistical rms noise of $B_{\mathrm{gs}}^{* *}(x)$ per frame is given by:

$$
\Delta B_{\mathrm{gs}}^{* *}(x)=\left(a \sigma_{I}^{2}+1 / N_{\mathrm{ph}}\right) / \sqrt{M(x)}
$$

where $N_{\mathrm{ph}}$ is the mean number of photons per coherence area of the scintillation pattern (radius $r_{c}$ ) and $M(x)$ is the number of independent cells, i.e., the ratio of $P(x)$, the overlapping pupil area, to $\pi r_{c}^{2}$ the coherence area of the scintillation patterns. Following Vernin \& Azouit (1983), we take $r_{c}$ equal to the Fresnel radius of the turbulent layer which dominates the scintillation pattern (i.e., with the largest $\sigma_{I}^{2}(h)$ ). We then have 
$r_{c}=\sqrt{\lambda H_{0} / 2 \pi}$, by noting $H_{0}$, the distance of that layer to the plane of analysis, and:

$$
M(x)=2 P(x) /\left(\lambda H_{0}\right)
$$

When considering Eq. 6 at $x_{\text {left }}$, the center of the left satellite, we can estimate the uncertainty of the optical turbulence factor (cf. Eq. 3):

$$
\Delta J\left(x_{\text {left }}\right)=\frac{\Delta B_{\mathrm{gs}}^{* *}\left(x_{\text {left }}\right)}{K(0, H)}
$$

with $K(0, H)=b \mathcal{C}(0, H) * S(0)$. Using Eqs. 2, 9 and 10, it comes:

$$
\Delta J\left(x_{\text {left }}\right)=\frac{3.7010^{-2} \lambda^{5 / 3} H^{-5 / 6} H_{0}^{1 / 2}\left(a \sigma_{I}^{2}+1 / N_{\mathrm{ph}}\right)}{b \sqrt{P(x)}}
$$

This noise can be reduced by averaging the signal on $n$ independent frames and integrating it over the full area of the satellites. More generally, the total noise on the estimation of $J$ for given layer from the measurement of a triplet can then be approximated with:

$$
N_{J}(H) \approx \frac{\Delta J\left(x_{l e f t}\right)}{c_{\tau} \sqrt{n} \sqrt{H / H_{0}}}
$$

where $c_{\tau}$ is the attenuation factor affecting the signal of various origin such as the decorrelation due to the length of the integration time, the non-steadiness of the atmospheric turbulence and the deviations from Taylor's assumption. We have found experimentally that a typical value of $c_{\tau}$ is $\sim 0.7$. The term $\sqrt{H / H_{0}}$ takes into account the reduction of noise due to the possible integration of the signal on a disk of diameter $\Delta \mathcal{R}(h)$ (cf. Eq. 7 ).

\section{Automatic wind velocity measurements}

\subsection{Introduction}

From the previous section, we see that the basic problem to obtain wind velocity profiles from the cross-correlation function $C_{\mathrm{c}}^{* *}$ is to detect the triplets produced by the 
turbulent layers. For each triplet, the wind parameters (horizontal amplitude and direction) can be derived from the position of the central peak, whereas the altitude of the layer are computed from the distance between the two satellites.

The experimental data to process consists of images which are measurements of the 2-D spatio-temporal cross-correlation function $C_{\mathrm{c}}^{* *}(\mathbf{r}, \Delta t)$ (with $\Delta t=20 \mathrm{~ms}$ or $40 \mathrm{~ms}$ ). In the following we shall assume that they have been filtered out from experimental noise and rotated so that the lines ( $x$ axis, in the following) are parallel to the direction of separation of the binary, as those assumptions were true for the cross-correlation data that we have used to test our method.

\subsection{Principle of our method}

We have chosen to use an iterative approach based on the CLEAN algorithm, as we did for the inversion of $C_{\mathrm{N}}^{2}$ profiles for SCIDAR measurements (Prieur et al., 2001). We wanted to take advantage of the experience acquired with the interactive program developed by Avila et al. (2001), which had shown its efficiency in providing good measurements. With Avila's program, the peaks are removed from the cross-correlation function with successive steps. For each step, the location of the central peak and satellites of a triplet are entered by the user, with the mouse, and then removed from the cross-correlation function. The user performs as many iterations as necessary in order to obtain a final image without any detectable peaks.

The method we propose is an "automated version" of Avila's program. For each iteration, the image is scanned for searching a central peak and two satellites. The validity of this triplet is determined with an analysis of the morphology, the brightness of the peaks, and the scintillation variance expected at the corresponding altitude. When all criteria are 
satisfied, the parameters of this layer (altitude, wind velocity, and direction) are stored into a file, and this triplet is removed (i.e. "cleaned"). The resulting image will be called residual map, whereas the image built with the valid triplets will be called clean map. The program proceeds with successive iterations, in order to detect (and then remove) all the triplets that are associated with turbulent layers.

\subsection{Description of the algorithm}

Let's define the main objects that we shall use in this section. The cpeaks and the clusters, are associated with the central peaks and the satellites, respectively. The triplets are made of two clusters and one cpeak. The cclean will be the CLEAN components detected in the CLEAN process. In our current implementation in C, they are represented as "structures", with many fields to qualify them (position, intensity, size, etc). In object-oriented languages, they could be genuine "objects".

The algorithm is presented in Fig. 2. It proceeds in four steps:

\section{Step 1: Detection of the central peak}

We first determine the center of the central peak cpeak by looking for the maximum in the current residual map (which is initialized to the cross-correlation function when starting the program). Its intensity will be called cpeak.zcent. A Gaussian function is then fitted within a small region around that maximum, which allows a more precise determination of the location (cpeak.xcent, cpeak.ycent) of the center of cpeak, and thus of the velocity of the possible corresponding turbulent layer(s).

To allow for subsequent detection of the (fainter) satellites, the intensity of cpeak needs to be large enough. We use a threshold on cpeak.zcent of xsigma1 for validating 
this peak. A typical value for xsigma1 is $6 \sigma_{c}$, where $\sigma_{c}$ was the standard deviation of the background (i.e. area free of any triplets) of the cross-correlation function. The program stops when the residual map does not exhibit any maxima larger than this value.

\section{Step 2: Detection of CLEAN components (satellites)}

Then an iterative CLEAN process of the satellites is performed inside the horizontal strip centered on (cpeak.xcent, cpeak.ycent), with a width $\Delta \mathcal{R}$ (cf. Eq. 7). For each $j$ th iteration, the location of the pixel with the maximum intensity is searched for. Its coordinates are noted $(\operatorname{cclean}[j] . x, c c l e a n[j] . y)$, and its intensity $c c l e a n[j] . z$. Then the function $\alpha \times \mathcal{C}(\mathbf{r}, H) * S(\mathbf{r})$ centered on $(\operatorname{cclean}[j] . \mathrm{x}, \operatorname{cclean}[j] . \mathrm{y})$, is subtracted from the current residual map to "clean" this central peak (cf. Sect 2). Like in most implementations of the CLEAN method, only a fraction of the maximum is "cleaned" at each iteration (we use $\alpha=0.30$ ). The value of $H$, which is needed to compute $\mathcal{C}(\mathbf{r}, H)$ is derived from the distance from cclean $[j]$ to cpeak (cf. Sect. 2). The iterative process on $j$ stops when the noise level is reached, i.e. $\operatorname{cclean}[j] . z<x$ sigma2, or when the number of CLEAN components exceeds a certain limit j_max. A typical value for xsigma2 is $3 \sigma_{c}$. The test on $j$ max is needed for safety to avoid problems when xsigma2 has been set too small.

\section{Step 3: Gathering CLEAN components into triplets}

A morphological analysis is then performed on the set of the CLEAN components $c c l e a n[j]$ detected in step 2. The purpose of this analysis is to sort out those numerous components and select those who belong to possible satellites associated with the central peak cpeak. This is done in two steps:

- The routine group_to_clusters groups the components cclean[j] into clusters, that will be candidates for satellites associated with turbulent layers, by performing 
a morphological analysis. This routine proceeds with successive iterations. First, the density of neighbours within $\mathcal{R}(h)$ (Eq. 7) is computed for each CLEAN component. The component having the maximum density of neighbours is taken as the center of a new cluster, and its neighbours (within $\mathcal{R}(h)$ ) are associated with this cluster. The components belonging to this cluster are then neutralized for the next iterations. The routine stops when the maximum neighbour density is too small (typically less than $3)$.

- The routine make_triplets performs an analysis of those clusters in order to select the pairs of clusters that will be good candidates for satellites of cpeak linked to a turbulent layer. When such a pair is found it constitutes a triplet. The criteria used here are morphological only: two clusters located on the left and on the right of the central peak cpeak, respectively, form a new triplet when their distances to that peak are similar. At this stage, it is also possible to detect some "foreign peaks", i.e. possible central peaks (and their satellites) generated by turbulent layers that have different velocities from that of the layer(s) associated with the central peak cpeak (see examples in Sect. 4.1). Indeed, a foreign central peak can be characterized as a bright cluster without any counterpart on the other side (left/right) relative to the central peak cpeak, with some satellites located symmetrically relative to it. Foreign peaks with their corresponding satellites are then processed separately.

At the end of this analysis, all the components $c c l e a n[j]$ that do not belong to clusters that have been grouped into triplets are removed from the CLEAN map, and the residual map is updated accordingly.

\section{Step 4: Testing the validity of the triplets}

Two tests of validity are then performed for each triplet: (loop on it index in Fig. 2). 
- check_symmetry: a thorough analysis of the symmetry of the two clusters belonging to the triplet relative to the central peak is performed. For the morphology, we check that the variances in $x$ and $y$ of the left/right distributions of the cclean components are similar, and that the mean values for $y$ on both sides are also similar. Concerning the intensities, both clusters should have a comparable number of cclean components, and the intensity of the central peak should be larger than that of the satellites (cf. Sect. 2). Here the main difficulty is a possible contamination by a foreign peak which would have not been detected by the routine make_triplet.

- check_altitude: a final test of the validity of the clusters is done using the $C_{\mathrm{N}}^{2}$ profile. The selected clusters should correspond to an altitude $H$ where the scintillation variance, $\sigma_{I}^{2}(H)$, is larger than the threshold xsigma2 used for detecting the satellites in the cross-correlation function. Note that the profile of the scintillation variance is derived from the $C_{\mathrm{N}}^{2}$ profile using Eq. 2 .

In the case of "multiple layers", i.e. turbulence layers with similar wind velocities and different altitudes, two or more triplets are associated with the central peak cpeak, and the layer index klayer is increased accordingly. Actually, in the current implementation of this program, a more precise determination of the location of the central peak is performed for each valid triplet, taking into account the location of the two satellites. This allow us to disentangle the cases when multiple layers have their central peaks superimposed.

Each time a valid triplet is found, the central peak is "cleaned" using the information contained in the satellites. The program removes from that peak $\frac{a \alpha}{b} \mathcal{C}(\mathbf{r}, H) * S(\mathbf{r})$ (see Eqs. 1 and 5). At the end of the process, there may remain some non-negligible residuals at this location, since the satellites are not always fully detected, due to the presence of noise. Thus, to allow the algorithm to converge, all pixels belonging to the disk centered on (cpeak.xcent, cpeak.ycent) with a diameter $\Delta \mathcal{R}$ are invalidated for further search of 
central peaks in the residual map.

When a triplet is rejected during this analysis, all CLEAN components belonging to its two clusters are removed from the CLEAN map, and the residual map is updated accordingly.

\section{General remarks:}

Note that all the CLEAN components that are not associated with satellites of valid triplets are restored to the residual map. They are thus available for another processing in subsequent iterations. This allows successful analysis of complex cases (e.g., examples of Sect. 4.1).

The sensitivity of the algorithm can be easily tuned by changing the values of the two thresholds xsigma1 and xsigma2 that are used to stop the search for the central peak and the satellites, respectively.

An option for processing structures with a single satellite is also possible. This may be needed when the binary star is widely separated and/or when the wind velocities are large: one of the satellites may fall out of the cross-correlation map. In this case, the tests about symmetry are invalidated, and the only remaining test is that performed by check_altitude with the $\sigma_{I}^{2}$ profile. To improve the stability, and avoid a possible avalanche of spurious detections, the thresholds xsigma1 and xsigma2 may then need to be enlarged, i.e. the central peak and its satellite must have a better signal-to-noise ratio than when full triplets are detected. 


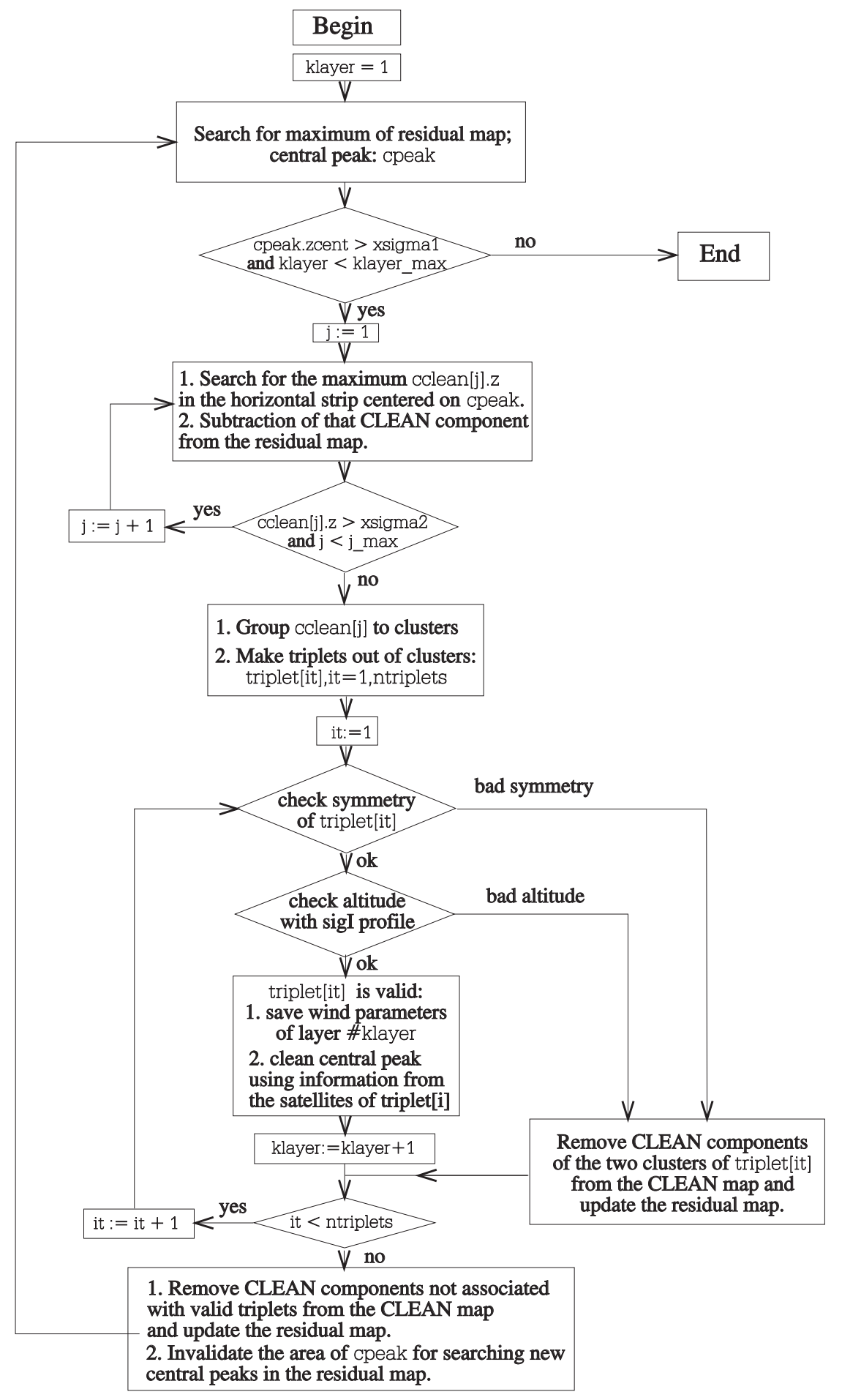

Fig. 2.- Algorithm of our method for computing wind profiles. 


\section{Analysis of the results}

\subsection{Examples of processing}

We present here some examples to illustrate the processing by our program of some typical cases.

\section{Multiple layers}

The detection of layers with similar velocities is rather difficult because the corresponding central peaks are superimposed. The triplets 1 and 2 of Fig.3.A illustrate this case. Moreover, the left satellite of triplet 1 is mixed with the central peak of triplet 5 . Despite those two difficulties, the program identified well each layer. It is an example of "multiple layers" (cf. Step 4 of Sect. 3.3). The pairs of clusters corresponding to the two layers 1 and 2 located at different altitudes are grouped to the same central peak to form two triplets. After validation of those triplets, the accurate location of the central peaks is then set to the mean of the corresponding satellites centers.

\section{Thick layer}

Rather often, the data show comma-like structures, which correspond to a series of layers at similar altitudes (a so-called "thick layer") and significantly different velocities. An example is presented in Fig. 3.Bb. The program finds four layers in the "comma" structure on the bottom-left corner of the cross-correlation maps. As can be seen in Fig. 3.Ba layers 3, 5 and 6 are located at the same altitude whereas layer 4 is slightly higher. Indeed when the altitude differences are smaller than the GS altitude resolution $\Delta \mathcal{R}(h) / \boldsymbol{\rho}$ (cf. Eq. 7), the same (mean) altitude is attributed to those layers.

\section{Mixed-up case}


Figs. 3.Cb illustrates the case of a particularly difficult situation when triplets are mixed up along the same line (here the triplets 2 and 3). This figure shows that the program is able to handle such situations. As explained in Sect. 3.3, this can be done in two ways: either by identifying a "foreign" central peak and its satellites during the processing of the brightest (and first detected) central peak, or by rejecting all the clusters not associated with the brightest central peak during this processing and by a specific processing of the "foreign" triplet during the subsequent iteration which starts by the detection of the central foreign peak.

\section{Wind velocity at the ground level}

When the separation of the double star and/or the analysis-plane distance from the pupil $\left(h_{\mathrm{gs}}\right)$ are not large enough, the turbulence near the ground can produce triplets with satellites partially superimposed on the central peak.

This is the case of layers 1 and 2 in Fig. 3.Bb and layer 1 in Fig. 3.Cb. As can be seen, the program handles well this complexity. For altitudes close to the ground level, the program works with the concept of "pattern recognition". The clusters corresponding to the satellites are identified by a thorough analysis of the intensity profile around the central peak.

The detection of the wind at the level of the ground is a very important feature because it enables us to determine the $C_{\mathrm{N}}^{2}$ inside the telescope dome. As explained by Avila et al. (2001), when two layers are detected at ground level with zero and non-zero velocities, respectively, then the first layer can be attributed to the turbulence inside the dome. 

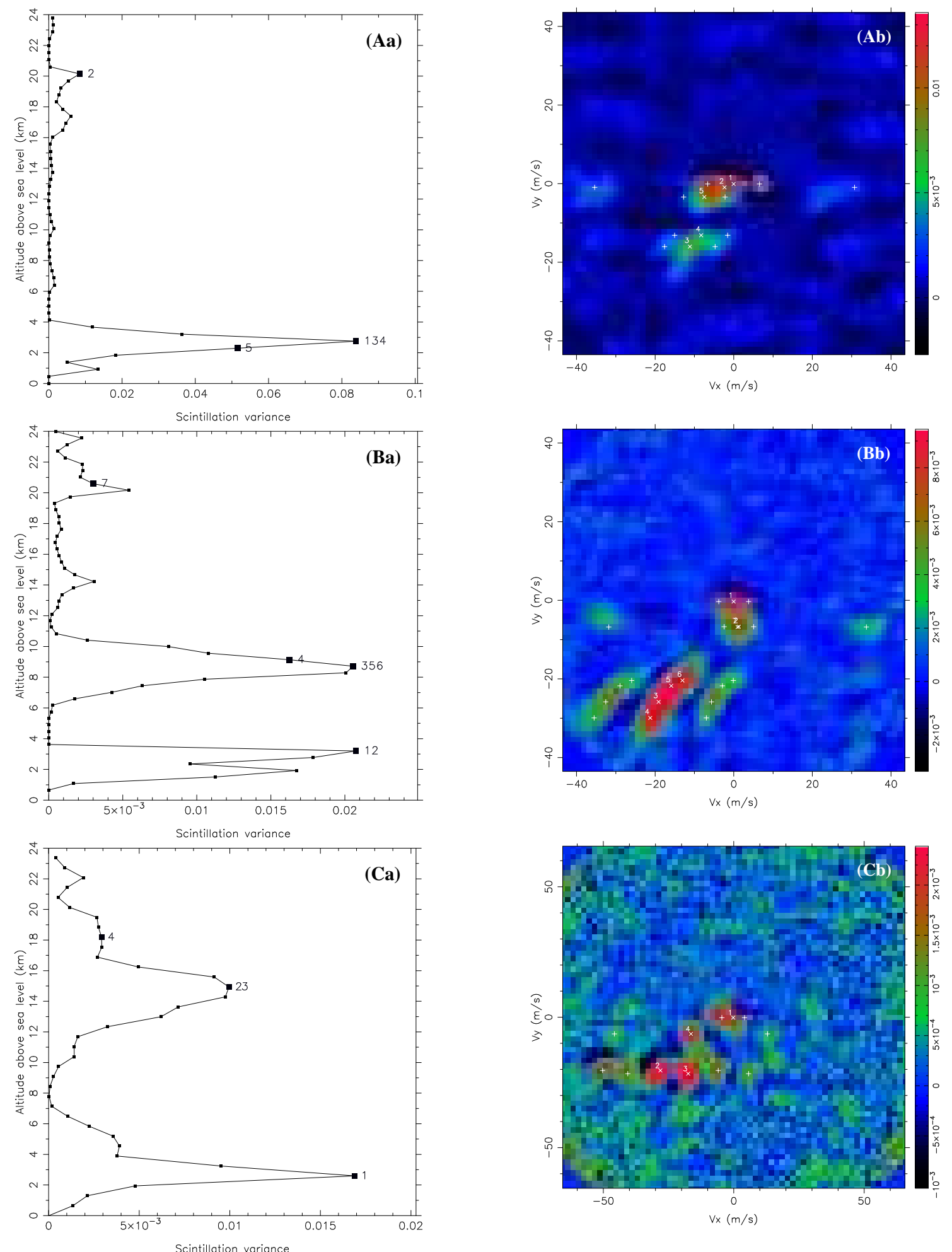

Fig. 3.- Examples of processing. From top to bottom: A. multiple layers with similar velocities; B. thick layer with a velocity gradient; C. superimposed triplets. Left (a): Scintillation variance profile. Right (b): cross-correlation function with crosses on the detected peaks: " $\times$ " for central peaks, and "+" for satellites. 


\subsection{Comparison with interactive processing}

In this section, we compare the results derived with this method to those obtained by R. Avila with the "interactive" data reduction. We have used the campaign of GS observations performed in 2000 with the $2.1 \mathrm{~m}$ telescope of San Pedro Mártir (SPM), which are described in Avila et al. (2004).

In Figs $4 \mathrm{a}$ and 4b, we have displayed the velocity profiles obtained for the night of May 19th 2000 with the automatic and interactive programs, respectively. In this example, a good compromise between sensitivity and robustness was obtained with xsigma1 $\sim 7$ and xsigma $2 \sim 2.4$. For each observation, we have plotted a black dot at altitude zero, when the turbulence inside the dome (with zero velocity) was detected. It thus clearly appears that our program detects very well the turbulence layers close to the level of the ground, and even inside the dome. Furthermore, the detection rates in automatic and interactive modes are very similar.

The $C_{N}^{2}$ profiles measured during that night are displayed in Fig. 4c. This night can be considered as typical with the highest velocity winds of around $30 \mathrm{~m} . \mathrm{s}^{-1}$ for the turbulent layers at high altitude in the range $10-15 \mathrm{~km}$. In this plot, the altitude of $2800 \mathrm{~m}$ of SPM observatory is indicated with a white line.

Figs $4 \mathrm{a}$ and $4 \mathrm{~b}$ show that the results obtained by the interactive and automatic modes are in very good agreement, especially for the altitudes with a $C_{N}^{2}$ with a good signal-to-noise ratio. Indeed, on the files we have processed, the main differences between the two modes were found for the altitudes with a small level of turbulence, which correspond to a small signal-to-noise ratio (SNR) of the $C_{N}^{2}$ profiles. But there also remains some particularly difficult situations with significant differences between interactive and automatic procedures although the SNR is good. The program (and humans) may not detect all the layers and/or give false detections. This rate of false detection is dependent on the complexity of the 
situations and on the values of xsigma1 and xsigma2, which tune the sensitivity.

Of course this program is not perfect. Despite all our efforts, it cannot integrate all the wisdom of human brain. There will always remain some situations when a skilled user will be superior to this program, and will detect some turbulent layers with a lower SNR or better disentangle particularly complex cases. The CLEAN-based process we have chosen is versatile, and allows a full integration of the automatic and the interactive modes. In the current version of our program, the user can add or remove triplets interactively on the results obtained with the automatic mode. For desperate cases, the user can even process everything in the interactive mode only.

We can quantify the level of detection with the filling factor, which is defined as the ratio of the sum of $J(h)$ for the detected layers over the total sum of $J(h)$ for all layers. In Fig. 5a, we have displayed the filling factor versus the time of observation for the measurements of the 19th May. In this figure, one can see that the automatic and interactive procedure lead to very similar filling factors, which indicates that the detection levels are nearly the same in both cases. The parameters that can be derived from the two sets of measurements are also very similar. Fig. $5 \mathrm{~b}$ shows an example of $\tau_{\mathrm{AO}}$, the coherence time for full-correction adaptive optics, derived from the turbulence and wind velocity using (Roddier, Gilli, \& Lund, 1982):

$$
\tau_{\mathrm{AO}}=0.519\left(\frac{2 \pi}{\lambda}\right)^{-6 / 5}\left[\int \mathrm{d} h|\mathbf{V}(h)|^{5 / 3} C_{\mathrm{N}}^{2}(h)\right]^{-3 / 5}
$$

The performances of both the interactive and automatic procedures can also be compared with the theoretical expectations using the noise estimation provided by Eq. 13 . In Fig. 6, we have displayed the $J$ amplitudes of the detected layers and the theoretical curves corresponding to $\mathrm{SNR}=3$ for the two binaries $\zeta$ Crb and 95 Her observed during the night of May 19th 2000 (with $h_{\mathrm{sg}}=-4 \mathrm{~km}, \sigma_{I}^{2} \sim 0.34, n=2000, H_{0}=13 \mathrm{~km}$, and $N_{\mathrm{ph}} \sim 100$ and 120 photons for $\zeta$ Crb and 95 Her, respectively). This figure shows that the 
detectivities of the automatic and interactive procedures are very similar, and are close to the 3- $\sigma$ level.

\section{Conclusion}

Our CLEAN-based method was implemented and successfully tested on extensive data from GS observations made in San Pedro Mártir in 2000. The wind velocity parameters (velocity and direction) derived with this method are fully compatible with the results obtained with interactive programs.

This method allows an automatic determination of the wind parameters in altitude, which, combined with the $C_{\mathrm{N}}^{2}$ profiles, provides a full characterization of the turbulence above the site of observation. in a non-supervised mode. This opens the possibility of processing large amounts of data, and even doing real-time processing. Adaptive optics systems working on telescopes located on the same site could then take advantage of the knowledge of the turbulence parameters for a better efficiency.

Acknowledgments: This work is based on observations made with the $2.1 \mathrm{~m}$ of San Pedro Mártir (Mexico). Funding for the measurement campaign was provided by grants J32412E from CONACyT, IN118199 from DGAPA-UNAM, M97U01 from ECOS-ANUIES and the TIM project (IA-UNAM). 

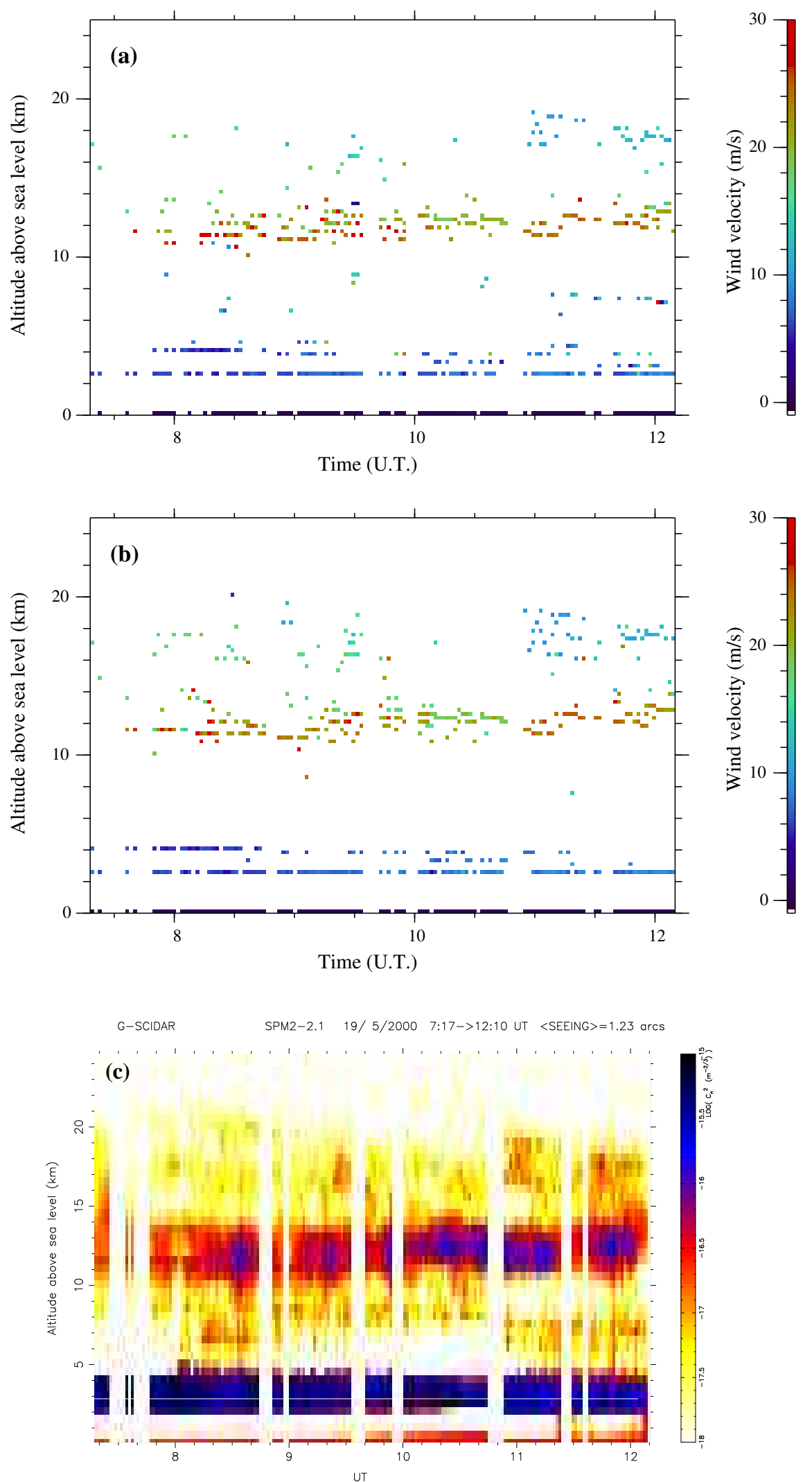

Fig. 4.- Vertical wind velocity profiles measured with automatic (a), and interactive (b) procedures. The corresponding $C_{N}^{2}$ profile is displayed in (c) (night of 19/05/2000). 

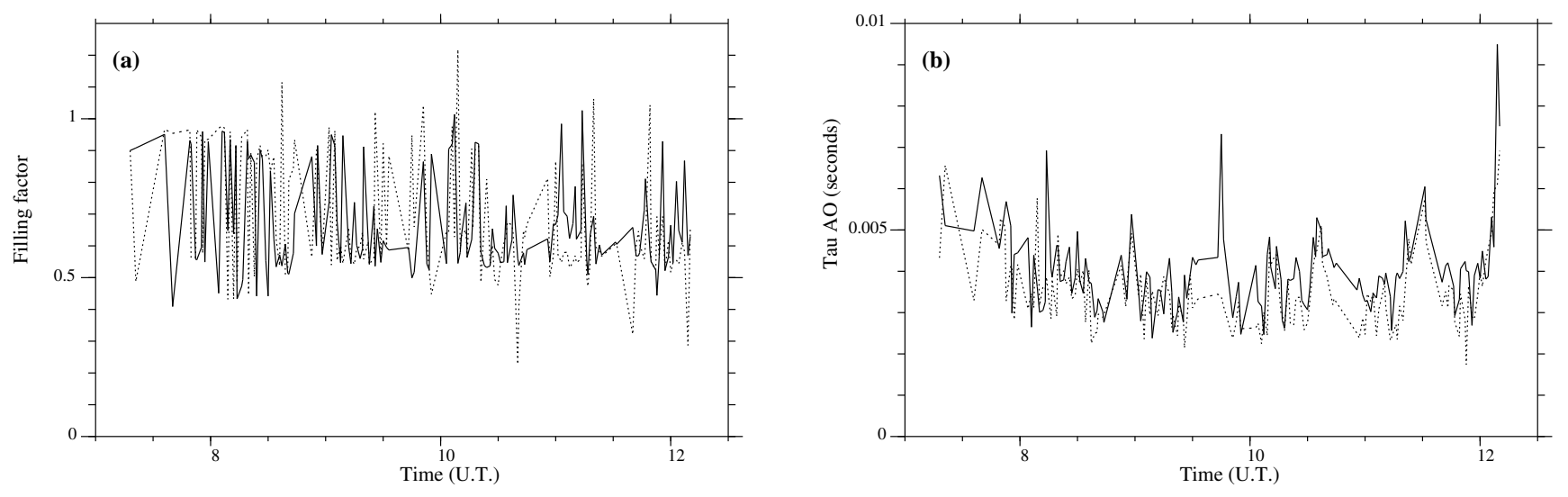

Fig. 5.- Filling factor (a), and $\tau_{\mathrm{AO}}$ (b) obtained with automatic (solid line) and interactive (dashed line) procedures, versus the time of observation (night of 19/05/2000). 

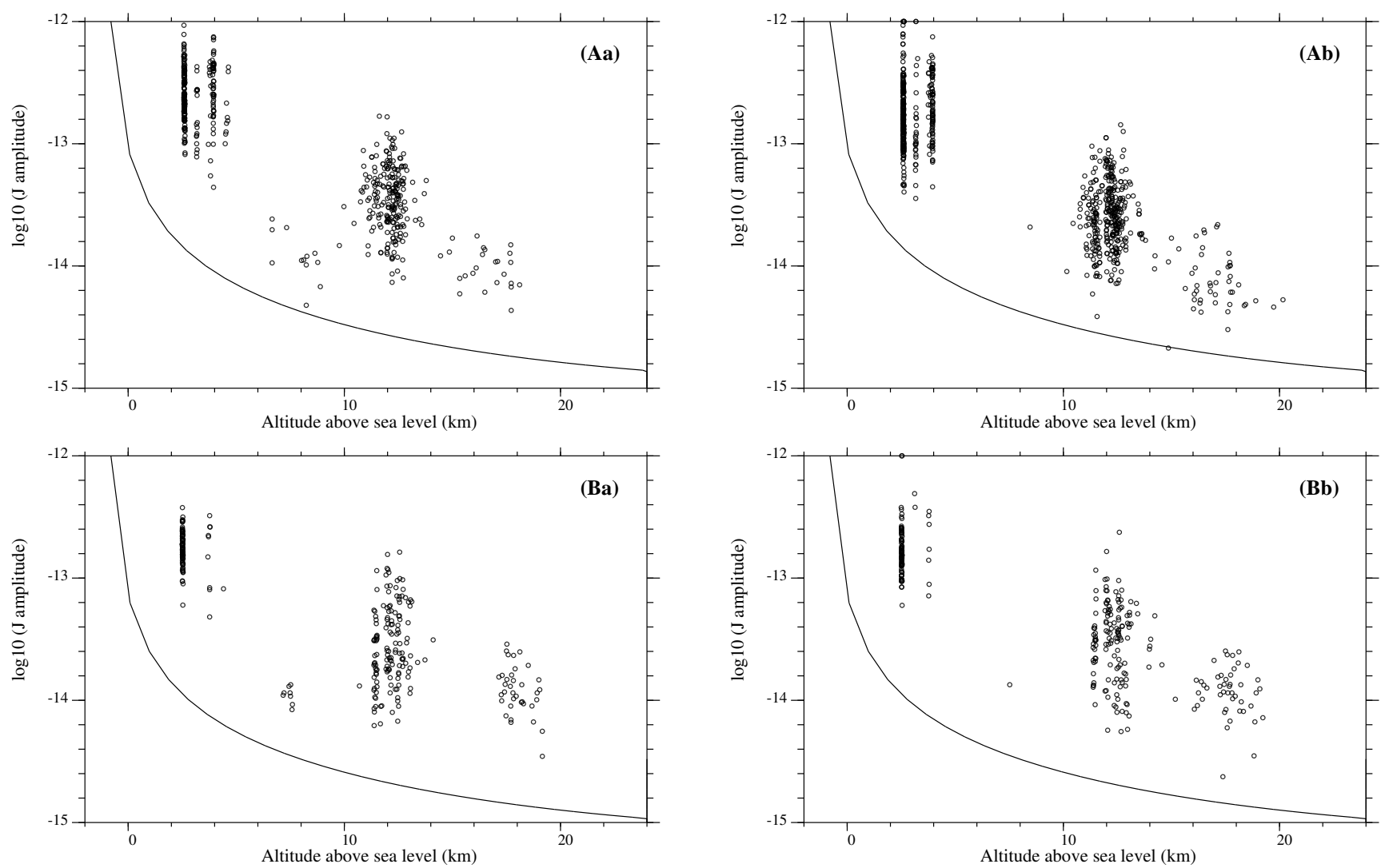

Fig. 6.- Optical turbulence factors of the layers detected with automatic (a) and interactive (b) procedures for $\zeta$ Crb (A) and 95 Her (B) (open circles). The theoretical detection curve corresponding to $\mathrm{SNR}=3$ is plotted as a solid line (night of $19 / 05 / 2000$ ). 


\section{REFERENCES}

Avila, R., Vernin, J., \& Masciadri, E. 1997, Appl. Opt. 36, 7898

Avila, R., Vernin, J., \& Cuevas, S. 1998, PASP 110, 1106

Avila, R., Vernin, J., \& Sánchez, L.J. 2001, A\&A, 369, 364

Avila, R., et al. 2004, RevMexAA (SC), 19, 11

Caccia, J. L., Azouit, M., \& Vernin, J. 1987, Appl. Opt. 26, 1288

Chun, M., et al. 2002, http://kolea.ifa.hawaii.edu/ mchun/mkseeing/MKSCHome.html

Fuchs, A., Tallon, M., \& Vernin, J. 1998, PASP 110, 86

Klückers, V.A., Wooder, N.J., Nicholls, T.W., Adcock, M.J., Munro, I., \& Dainty, J.C. 1998, A\&AS 130, 141

McKenna, D., et al. 2003, Proc SPIE, 4839, 825

Rocca, A., Roddier, F., \& Vernin, J. 1974, J. Opt. Soc. Am., 64, 1000

Prieur, J.-L., Daigne, G., \& Avila, R. 2001, A\&A, 371, 366

Roddier, F. 1981, Prog. Opt., 19, 281

Roddier, F., Gilli, J., \& Lund, G. 1982, J. Opt. (Paris), 13, 263

Tokovinin, A.A. 1997, VLT-TRE-UNI-17416-0003 (ESO, Garching)

Vernin, J. 1992, in Wave propagation in Random Media (Scintillation), Ed. V.I. Tatarskii, A. Ishimaru, \& V.U. Zavorotny, SPIE-ISOE, Seattle, Washington, 248

Vernin, J., \& Azouit, M. 1983, J. Opt. (Paris), 14, 131 
Vernin, J., et al. 2000, 1998 Gemini Site Testing Campaign at Cerro Pachón and Cerro Tololo, AURA Report 